\title{
Sarcomas other than Kaposi's sarcoma in HIV
}

\author{
S Mohammedali MBChB, MMed(Surg), HDipOrth SA \\ LC Marais MBChB FC(Orth)SA, MMed(Ortho), CIME, PhD \\ University of KwaZulu-Natal, South Africa \\ Corresponding author: \\ Dr Mohammedali Shamshudin \\ Department of Surgery \\ The Aga Khan University Hospital \\ Nairobi, Kenya \\ PO Box 030270-00100, Nairobi, Kenya \\ Tel: +254711092115 \\ Fax: +254203743935 \\ Email: shamshuza@yahoo.com
}

Department of Orthopaedics, University of KwaZulu-Natal, Pietermaritzburg, South Africa

Tumour Sepsis and Reconstruction Unit, Department of Orthopaedics, School of Clinical Medicine,

\begin{abstract}
Introduction: Kaposi's sarcoma and lymphoma are some of the malignancies known to be associated with HIV infection and afflict many people living with HIV/Aids, as published widely in previous literature. In contrast, scarce information is available with regard to the occurrence of other primary musculoskeletal malignancies and whether there may be a causal relation between the occurrence of these malignancies and HIV. The aim of this study is to describe which sarcomas (other than Kaposi's) occur in patients living with HIV.

Methods: A retrospective chart review was performed of consecutive adult individuals who presented to our tumour unit with musculoskeletal sarcomas other than Kaposi's sarcoma. The histological diagnosis of tumours in HIV-positive patients were then compared to that of an age-matched cohort of HIV-negative patients.

Results: A total of 59 patients were included in the study. Thirty patients were positive and 29 patients negative. We recorded a wider variety of sarcomas in HIV-positive individuals than previously reported. There was a tendency towards an increased number of cases of rhabdomyosarcoma in the HIV-positive group and chondrosarcoma in the HIV-negative group although this did not reach statistical significance, $p=0.05$ and 0.08 respectively. There was no difference in the prevalence of metastasis at the time of presentation with 16 cases having metastases in the HIV-positive group $(53.3 \%)$ and 14 cases $(48.2 \%)$ in the HIV-negative group, $p=0.69$.

Conclusion: The prevalence of musculoskeletal sarcomas other than Kaposi's sarcoma in HIV-positive patients and a tendency towards an increased prevalence of rhabdomyosarcoma requires further investigation. Long-term studies to assess the influence of antiretroviral therapy on the prevalence and prognosis of these sarcomas are required.
\end{abstract}

Key words: HIV, non-Kaposi's sarcoma, musculoskeletal, non-Aids defining cancers (NADCs)

http:/ / dx.doi.org/10.17159/2309-8309/2017/v16n1a5

\section{Introduction}

HIV / Aids is an immune-compromising disease that may be accompanied by the development of opportunistic infections and malignancies. There is a high prevalence of HIV infection in South Africa with an estimated 6.8 million cases in 2014. ${ }^{1}$ In the province of KwaZulu-Natal, where this research was performed, an estimated $21.5 \%$ of adults are living with HIV. ${ }^{2}$ Significant strides have been made in terms of the management of HIV, and near-normal life expectancy can be expected if anti-retroviral therapy is timeously instituted. ${ }^{3}$

Cancers can be categorised by their associations with $\mathrm{HIV}$ / Aids as being Aids-defining cancers (ADCs) such as Kaposi's sarcoma, non-Hodgkin's lymphoma and cervical cancer, which are all virus-related, and non-Aids-defining cancers (NADCs) (Figures 1 and 2). ${ }^{4}$ The overall prevalence of cancers in HIV has declined following the introduction of anti-retroviral therapy but recent literature suggests that there may still be an increase in non-virus-related NADCs. ${ }^{5}$ 

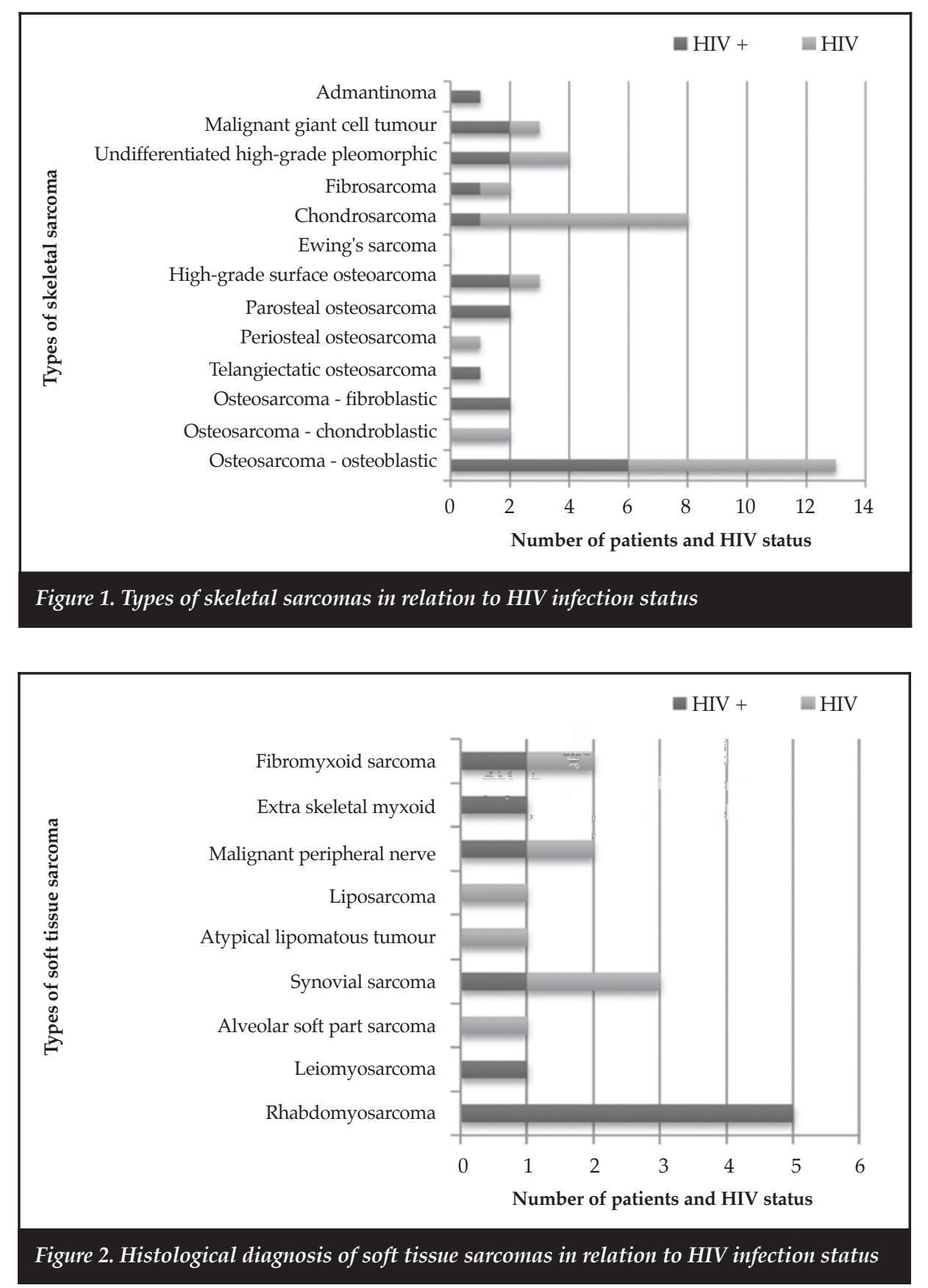

Sarcomas are malignant tumours that originate from connective or non-epithelial tissue. They are rare and account for less than $1 \%$ of all malignancies diagnosed. There are no locally published statistics on prevalence or incidence. While the majority of information about malignancies and HIV comes from high-income countries, 90\% of people living with HIV reside in sub-Saharan Africa. ${ }^{6}$ There is a plethora of studies that associate Kaposi's sarcoma (KS) with HIV; in fact, it is an Aids-defining malignancy but there is a paucity of literature on other limb and girdle sarcomas. ${ }^{5}$ Not much information has been published about the prevalence of non-Kaposi's sarcomas that afflict the musculoskeletal system in people living with HIV/Aids. Most of the published work has concentrated on KS and lymphoma, which are the more common malignancies associated with HIV infection. Other sarcomas are typically classified under the non-Aids-defining cancer group that are not specifically defined in large studies investigating the prevalence or incidence of cancer in HIV patients. ${ }^{7-9}$ 


\section{Methods}

\section{Study design}

We retrospectively reviewed the charts of all adult patients who were treated for sarcoma at our tumour unit from January 2011 to December 2015. Eligible patients were identified from a prospectively gathered database. Patients were included in the study if they were 18 years or older and there was a histologically confirmed diagnosis of sarcoma involving soft tissue and/or bone. The exclusion criteria were: patients whose clinical record was incomplete or in whom the histologic diagnosis was inconclusive for sarcoma; and patients with the diagnosis of lymphoma, myeloma or metastatic bone disease. The subjects' charts were reviewed and data extracted in order to describe the patient demographics, location of the tumour, histological diagnosis, HIV status and the presence of metastases.

Table I: Patient demographics and distribution of bone and soft tissue sarcomas in HIV-positive and -negative patient groups

\begin{tabular}{|c|c|c|c|}
\hline Characteristic & $\begin{array}{c}\text { HIV-positive } \\
\text { n }(\%)\end{array}$ & $\begin{array}{c}\text { HIV-negative } \\
\text { n (\%) }\end{array}$ & $p$-value \\
\hline \multicolumn{4}{|l|}{ Age } \\
\hline Mean (SD) & $37.5(11.58)$ & $43.4(16.75)$ & $0.11^{*}$ \\
\hline \multicolumn{4}{|l|}{ Gender } \\
\hline Male & 14 & 16 & \\
\hline \multicolumn{4}{|l|}{ Metastases } \\
\hline Present & 16 & 14 & $0.69^{* *}$ \\
\hline Bone tumours & 20 & 20 & $0.85^{\star *}$ \\
\hline Femur & 9 & 8 & \\
\hline Tibia & 6 & 6 & \\
\hline Fibula & 0 & 1 & \\
\hline Pelvis & 1 & 2 & \\
\hline Humerus & 4 & 1 & \\
\hline Ulna & 0 & 1 & \\
\hline Radius & 0 & 1 & \\
\hline Soft tissue tumours & 10 & 9 & $0.85^{\star *}$ \\
\hline Thigh & 6 & 2 & \\
\hline Pelvis & 3 & 0 & \\
\hline Leg & 0 & 2 & \\
\hline Foot & 1 & 1 & \\
\hline Knee & 0 & 2 & \\
\hline Arm & 0 & 2 & \\
\hline
\end{tabular}

All patients underwent local and systemic staging. Local staging consisted of radiographs and a magnetic resonance imaging (MRI) scan of the affected limb segment. Systemic staging included workup for medical co-morbidities, laboratory investigations and computerised tomography (CT) scan of the chest and abdomen. Informed consent for HIV testing was requested from all patients. Histology was obtained by formal incisional biopsy, according to standard biopsy principles, in all cases. Diagnosis was subsequently confirmed by combined radiological and histological evaluation.

\section{Statistical methods}

Data were analysed using Stata 13.0 (StataCorp LP 2013. College Station, Texas). Continuous variables were summarised using mean and standard deviation values. Categorical variables were summarised using frequency tables. An unpaired t-test was used to assess the age of patients in relation to HIV status. The association between HIV status and the presence of metastases was tested using the chi-square test. Fisher's exact test (twotailed) was used to test the independence of tumour site and histology on HIV status. A $p$-value of $<0.05$ was considered statistically significant for all tests.

\section{Ethical considerations}

All research was conducted according to the ethical principles for medical research on human subjects as defined by the World Medical Association Declaration of Helsinki (amended at the WMA General Assembly, Seoul, October 2008).

Ethical approval for conducting this research was obtained from BREC (Biomedical Ethics Review Board) prior to starting data collection. All research participants' information was held strictly confidential.

\section{Results}

Sixty-one files were screened of which two were excluded due to missing HIV data. Therefore, 59 patients met the eligibility criteria. The HIV-positive group consisted of 30 patients and HIV-negative group of 29 patients. Patient demographics are provided in Table I. As an age-matched cohort, there was no difference in the age range and mean age between HIV-positive (mean: 37.5 years; range: $21-64$; SD [standard deviation] $=11.58$ ) and HIV-negative group (mean: 43.4 years; range: $21-76$, SD: 16.75) $(p=0.11)$. There was an equal distribution of males and females. The distribution of tumours according to site is provided in Table I.

There was no significant difference in the rate of metastases at time of presentation, with $53.3 \%$ of patients in the HIV-positive group presenting with metastases compared to $48.2 \%$ in the HIV-negative group $(p=0.69)$. 
With regard to the type of sarcomas, the majority of patients in both groups were diagnosed with bone sarcomas, $20(66.7 \%)$ HIV-positive and $23(79.3 \%)$ HIVnegative. Osteogenic sarcoma was the most common bone sarcoma, $13(43.3 \%)$ positive and $11(37.9 \%)$ negative. Of these, conventional (osteoblastic) osteosarcoma numbered the highest, six $(20 \%)$ in the positive and seven $(24.1 \%)$ in the negative group. There was a tendency towards chondrosarcoma being more common in the HIV-negative group, seven $(24.1 \%$ ) as compared to one $(3.3 \%)$ in the HIV-positive group, but this difference did not reach statistical significance $(p=0.08)$.

Of the soft tissue sarcoma type, the overall numbers were similar, with ten $(33.3 \%)$ cases in the HIV-positive group vs six $(20.6 \%)$ in the HIV-negative group. However, it was observed that there were more cases of rhabdomyosarcoma in the HIV-positive group, five $(16.6 \%)$ HIV-positive vs zero (0\%) HIV-negative, $p=0.05)$.

\section{Discussion}

The aim of this study was to describe the histological types of sarcomas that can occur in patients living with HIV and to compare it to an age-matched HIV-negative cohort over the same period. At our institution, we had noted a wider variety of tumours that had not previously been published, and therefore instituted this study to document the various sarcomas that can occur in HIV. In our cohort of patients older than 18 years, osteosarcoma was the most common diagnosis, with no significant difference between HIV-positive and -negative patients $(p=0.35)$. While chondrosarcoma was more common in HIV-negative patients, the difference was insignificant $(p=0.08)$. Rhabdomyosarcoma was more common in HIVpositive patients $(p=0.05)$.

The immunological basis of the development of malignancies in HIV-infected individuals is not yet completely understood. One direct mechanism whereby retroviruses can cause cancer is through oncogene transduction. This mechanism has, however, only been seen in simple retroviruses and not with complex retroviruses like HIV. Many retroviruses do not possess viral oncogenes, but initiate tumour formation through the integration of proviral DNA near normal cellular proto-oncogenes, thereby activating their expression, by a mechanism termed 'proviral insertional mutagenesis'. Expression of c-myc proto-oncogene has been shown to be induced during HIV infection. ${ }^{10}$ Activation of c-myc oncogene has also been described in Aids-associated lymphoma. ${ }^{11}$ Furthermore the importance of the c-myc oncogene has been firmly established in the pathogenesis of certain sarcomas. ${ }^{12}$ These factors suggest that HIV infection may be related to the development of sarcomas.

The reported occurrence of sarcomas in the NADCs group is rare. Moreover, musculoskeletal sarcomas are rarely mentioned separately in NADC reports. ${ }^{13}$
Newnham et al. reported five soft tissue sarcomas and one bone sarcoma with the SIR (standardised incidence ratio) for soft tissue tumours being 3.6 and for skeletal malignancies 1.5. The specific histology of these sarcomas and their site was unfortunately not described, and it remains unclear if these included any of the sarcomas that we reported in our study. ${ }^{13} \mathrm{~A}$ study by Bhatia et al. identified a total of 176 non-Kaposi's sarcomas in immune compromised patients in the SEER database, 75 in people with HIV / Aids and 101 in transplant recipients. Leiomyosarcomas $(n=101)$ were the most frequently reported sarcomas, followed by angiosarcomas $(n=23)$ and fibrohistiocytic tumours $(\mathrm{n}=17) .{ }^{14}$ Leiomyosarcomas, angiosarcomas, osteosarcoma and fibrohistiocytic tumours are the only musculoskeletal malignancies previously associated with HIV. ${ }^{14-17}$ In our study, we noted a wider variety of cancers than any other prior study (Table II). There was a tendency to an increased number of cases of rhabdomyosarcoma in the HIV-positive group and chondrosarcoma in the HIVnegative group but this was not statistically significant.

Individuals with sarcoma who have concomitant HIV infection tend to fare worse than HIV-negative individuals both in terms of poor response to chemotherapy and death due to distant spread rather than HIV related..$^{15}$ In our study, we noted that the presence of metastasis at presentation was equal among HIV-positive and negative groups. This was in contrast to the American HIV/Aids Cancer Match Study (HACM). HACM data reported that $32.3 \%$ of people living with HIV with cancer present with distant disease versus $17.7 \%$ in the general population, $p<0.01 .^{18}$

The small number of cases in both groups is a shortcoming. The exclusion of adolescent patients (in whom osteosarcoma is most prevalent) further decreased the sample size which was too small, for example, to determine if there was an increased risk of metastases in any of the osteosarcoma subtypes. The retrospective nature of this study is a further limitation. We would have liked to follow these patients up prospectively to assess if they truly remained HIV-negative after recruitment into the study, and whether HIV influences the long-term outcome with concurrent sarcoma. We also did not assess for the usage of HIV treatment and its influence on outcomes. This study was also subject to referral bias as the database from which the information was retrieved comes from a single referral institution. The findings in this study may not be fully generalisable but our referral base and patient profile fit the majority in sub-Saharan Africa.

Our study reports a higher number and a wider variety of musculoskeletal sarcomas, other than Kaposi's, in HIV-positive patients compared to previous literature. Large prospective multicentre trials are required in our environment to assess this trend, their response to chemotherapy, surgery and long-term survival of this particular group of patients. 
Table II: Histological diagnosis according to HIV status of the patient

\begin{tabular}{|c|c|c|c|}
\hline Histology & $\begin{array}{l}\text { HIV-positive } \\
\text { n (\%) }\end{array}$ & $\begin{array}{c}\text { HIV-negative } \\
\text { n (\%) }\end{array}$ & $p$-value \\
\hline \multicolumn{4}{|l|}{ Bone } \\
\hline Osteosarcoma & & & $0.35^{*}$ \\
\hline \multicolumn{4}{|l|}{ Conventional osteosarcoma } \\
\hline Osteoblastic & $8(27 \%)$ & $7(24.1 \%)$ & \\
\hline Chondroblastic & $0(0 \%)$ & $2(6.9 \%)$ & \\
\hline Fibroblastic & $2(6.6 \%)$ & $0(0 \%)$ & \\
\hline Osteosarcoma variants & & & $0.42^{* *}$ \\
\hline Telangiectatic osteosarcoma & $1(3.3 \%)$ & $0(0 \%)$ & \\
\hline Periosteal osteosarcoma & $0(0 \%)$ & $1(3.4 \%)$ & \\
\hline Parosteal osteosarcoma & $2(6.6 \%)$ & $0(0 \%)$ & \\
\hline High-grade surface osteoarcoma & $2(6.6 \%)$ & $1(3.4 \%)$ & \\
\hline Ewing's sarcoma & $0(0 \%)$ & $0(0 \%)$ & 1.0 \\
\hline Chondrosarcoma & $1(3.3 \%)$ & $7(24.1 \%)$ & $0.08^{* *}$ \\
\hline Fibrosarcoma & $1(3.3 \%)$ & $1(3.4 \%)$ & 1.0 \\
\hline Undifferentiated high-grade pleomorphic sarcoma & $2(6.6 \%)$ & $2(6.9 \%)$ & 1.0 \\
\hline Malignant giant cell tumour & $0(0 \%)$ & $1(3.4 \%)$ & 0.49 \\
\hline Admantinoma & $1(3.3 \%)$ & $0(0 \%)$ & 1.0 \\
\hline \multicolumn{4}{|l|}{ Soft tissue } \\
\hline Rhabdomyosarcoma & $5(16.6 \%)$ & $0(0 \%)$ & $0.05^{* *}$ \\
\hline Leiomyosarcoma & $1(3.3 \%)$ & $0(0 \%)$ & 1.0 \\
\hline Alveolar soft part sarcoma & $0(0 \%)$ & $1(3.4 \%)$ & 0.49 \\
\hline Synovial sarcoma & $1(3.3 \%)$ & $2(6.9 \%)$ & 0.61 \\
\hline Liposarcoma & $0(0 \%)$ & $2(6.9 \%)$ & 0.24 \\
\hline Malignant peripheral nerve sheath tumour & $1(3.3 \%)$ & $1(3.4 \%)$ & 1.0 \\
\hline Extra skeletal myxoid chondrosarcoma & $1(3.3 \%)$ & $0(0 \%)$ & 0.49 \\
\hline \multirow[t]{2}{*}{ Fibromyxoid sarcoma } & $1(3.3 \%)$ & $1(3.4 \%)$ & 1.0 \\
\hline & $30(100 \%)$ & $29(100 \%)$ & \\
\hline
\end{tabular}

\section{Conclusion}

Patients living with HIV can develop a variety of musculoskeletal sarcomas other than KS. With universal access to antiretroviral therapy being legislated by many governments, these patients will live longer. Therefore, programmes for increased awareness among the medical and orthopaedic community and early referral to an appropriate centre will set the ground for future studies and eventually improved outcomes.

\section{Compliance with Ethics Guidelines}

\section{Ethical considerations}

All research was conducted according to the ethical principles for medical research on human subjects as defined by the World Medical Association Declaration of Helsinki (amended at the WMA General Assembly, Seoul, October 2008).

Ethical approval for conducting this research was obtained from BREC (Biomedical Ethics Review Board) prior to starting data collection. All research participants' information was held strictly confidential. 


\section{References}

1. UNAIDS. http://www.unaids.org/en/regionscountries/ countries/southafrica/ Accessed 13 January 2016.

2. Welz T, Hosegood V, Jaffar S, Batzing-Feigenbaum J, Herbst $\mathrm{K}$, Newell M. Continued very high prevalence of HIV infection in rural KwaZulu-Natal, South Africa: a population-based longitudinal study. AIDS 2007;21:1467-72.

3. Johnson LF, Mossong J, Dorrington RE, Schomaker M, Hoffmann CJ, et al. Life expectancies of south african adults starting antiretroviral treatment: collaborative analysis of cohort studies. PLoS Med 2013;10(4):e1001418. doi:10.1371/journal.pmed.1001418

4. Yanik EL, Napravnik S, Cole SR, Achenbach CJ, Gopal S, Olshan A, Dittmer DP, Kitahata MM, Mugavero MJ, Saag M, Moore RD, Mayer K, Mathews WC, Hunt PW, Rodriguez B, Eron JJ. Incidence and timing of cancer in HIV-infected individuals following initiation of combination antiretroviral therapy. Clin Infect Dis 2013 Sep;57(5):756-64.

5. Albini L, Calabresi A, Gotti D, Ferraresi A, Festa A, Donato F, Magoni M, Castelli F, Quiros-Roldan E. Burden of non-AIDS-defining and non-virus-related cancers among HIV-infected patients in the combined antiretroviral therapy era. AIDS Research and Human Retroviruses. Jul 2013;29(8):1097-104.

6. J.U.N.P.o.H.A., Global Report: UNAIDS report on the global AIDS epidemic 2013. Geneva, Switzerland.

7. Robbins HA, et al. Excess cancers among HIV-infected people in the United States. I Natl Cancer Inst. 2015;107(4):1-8.

8. Robbins HA, et al. Epidemiologic contributions to recent cancer trends among HIV-infected people in the United States. AIDS. 2014;28(6):881-90.

9. Bebawy SS, Netto JS, McGowan CC, Veloso VG, Engels EA, Sterling TR, Grinsztejn B. HIV and cancer: a comparative retrospective study of Brazilian and US clinical cohorts. *Infect Agent Cancer. 2015 Feb 2;10:4. doi: 10.1186/1750-9378-10-4. eCollection 2015.
10. Sun Y, Clark EA. Expression of the c-myc proto-oncogene is essential for HIV-1 infection in activated T cells. Journal of Experimental Medicine. 1999;189(9):1391-97.

11. Subar M, Neri A, Inghirami G, Knowles DM, Dalla-Favera $R$. Frequent c-myc oncogene activation and infrequent presence of Epstein-Barr virus genome in AIDS-associated lymphoma. Blood. 1988;72(2):667-71.

12. Broadhead ML, Clark JCM, Myers DE, et al. The molecular pathogenesis of osteosarcoma: a review. Sarcoma. 2011(1):1-12, Article ID 959248

13. Newnham A, Harris J, Evans HS, Evans BG, Moller H. The risk of cancer in HIV-infected people in southeast England: a cohort study. Br J Cancer 2005;92:194-200.

14. Bhatia K, Shiels MS, Berg A, Engels EA. Sarcomas other than Kaposi's sarcoma occurring in immunodeficiency: interpretations from a systematic literature review. Curr Opin Oncol. 2012 September;24(5):537-46.

15. Fizazi K, et al. Soft tissue sarcomas in HIV-infected adult patients. Eur J Cancer. 1996;32A(10):1812-14.

16. Chadwick EG, et al. Tumors of smooth-muscle origin in HIV-infected children. JAMA. 1990;263(23):3182-84.

17. Marais LC, Ferreira N. Osteosarcoma in Adult Patients Living with HIV/AIDS. ISRN Oncology. Volume 2013, Article ID 219369, 6 pages. Available from http:/ / dx.doi.org/10.1155/2013/219369

18. Coghill AE, et al. Elevated cancer-specific mortality among HIV-infected patients in the United States. J Clin Oncol. 2015;33(21):2376-83.

This article is also available online on the SAOA website (www.saoa.org.za) and the SciELO website (www.scielo.org.za). Follow the directions on the Contents page of this journal to access it. 